\title{
REMARKS ON GEOMETRIC PROPERTIES OF CERTAIN COEFFICIENT ESTIMATES
}

\author{
By Seiji KonaKaZawa
}

\section{Introduction.}

Let $\Sigma_{0}$ denote the class of functions, analytic and univalent in $|z|>1$ with the expansion

$$
f(z)=z+\sum_{n=1}^{\infty} b_{n} z^{-n}
$$

On some coefficient problems, extremal functions are odd or have real coefficients. Leung and Schober [5, Lemma 2.3] proved that the extremal function for the problem $\max _{\Sigma_{0}} \operatorname{Re}\left(b_{3}+\lambda b_{1}\right)$ must be odd. And Jenkins $[2, \S 6]$ presented a very simple proof of the inequality $\left|A_{3}\right| \leqq 3$ in the familiar class $S$ by showing that the extremal function for the problem $\max _{S} \operatorname{Re} A_{3}$ has real coefficients.

We represent such facts in terms of quadratic differentials by making use of Jenkins' General Coefficient Theorem. Then we give two applications. One is a simple proof of the fact that the third Ozawa number $B_{3}=3$ [1], [3] where $B_{3}=\inf \left\{t: \operatorname{Re}\left(t b_{1}-b_{3}\right) \leqq t\right.$ for all $\left.f \in \Sigma_{0}\right\}$ [6]. The other is the coefficient inequality for the coefficient functional $b_{3}+(1 / 2) b_{1}^{2}+\lambda b_{2}$ with real $\lambda$.

\section{Quadratic differentials.}

We use the following two special cases of Jenkins' General Coefficient Theorem (e.g. [7, Theorem 8.12]).

LEMMA 1.1. Let $\psi(w)=w+a_{1} w^{-1}+a_{2} w^{-2}+\cdots$ be univalent and admissible for the quadratic differential $Q(w) d w^{2}=\left(A_{0} w+A_{1}\right) d w^{2},\left(A_{0} \neq 0\right)$. Then

$$
\operatorname{Re}\left(A_{0} a_{2}+A_{1} a_{1}\right) \leqq 0 .
$$

If equality holds in (1.1), then $a_{1}=0$.

LEMMA 1.2. Let $\phi(w)=w+a_{1} w^{-1}+a_{2} w^{-2}+a_{3} w^{-3}+\cdots$ be univalent and admissible for the quadratic differential $Q(w) d w^{2}=\left(A_{0} w^{2}+A_{1} w+A_{2}\right) d w^{2}, \quad\left(A_{0} \neq 0\right)$.

Received January 8, 1987 
Then

$$
\operatorname{Re}\left(A_{0} a_{3}+A_{1} a_{2}+A_{2} a_{1}+\frac{1}{2} A_{0} a_{1}^{2}\right) \leqq 0 .
$$

If equality holds in (1.2), then $2 A_{0} a_{2}+A_{1} a_{1}=0$.

The lemma of Leung-Schober [5, Lemma 2.3] can be slightly generalized as follows.

THEOREM 1.3. Let $f(z)=z+\sum_{n=1}^{\infty} b_{n} z^{-n}$ be in $\Sigma_{0}$. If $\widehat{\boldsymbol{C}}-f(|z|>1)$ is on the trajectory arcs of the quadratic differential $Q(w) d w^{2}=\left(A_{0} w^{2}+A_{2}\right) d w^{2}, \quad\left(A_{0} \neq 0\right)$, then $f(z)$ is odd.

Since this can be proved by the same technique as theirs, we omit the proof.

COROLlary 1.4. Put the coefficient functional

$$
L(f)=\sum_{m=1}^{M} \alpha_{m} b_{1}^{m}+\left(\sum_{n=0}^{N} \beta_{n} b_{1}^{n}\right) b_{3},
$$

$f(z)=z+b_{1} z^{-1}+b_{2} z^{-2}+b_{3} z^{-3}+\cdots$, where $\alpha_{m}$ and $\beta_{n}$ are complex constants. Then

$$
\max _{\Sigma_{0}} \operatorname{Re} L(f)=\max _{f: \text { odd } \in \Sigma_{0}} \operatorname{Re} L(f) .
$$

Proof. Let $g(z)=z+\sum_{n=1}^{\infty} b_{n} z^{-n}$ be an extremal function for the problem $\max _{\Sigma_{0}} \operatorname{Re} L(f)$. If $\sum_{n=0}^{N} \beta_{n} b_{1}^{n}=0$, then $\max _{\Sigma_{0}} \operatorname{Re} L(f)=\operatorname{Re} L(g)=\operatorname{Re}\left(\sum_{m=1}^{M} \alpha_{m} b_{1}^{m}\right)$. Putting $\rho=\left|b_{1}\right|^{-1 / 2}$ and $\theta=-(1 / 2)$ arg $\left(b_{1}\right)$, we have $\rho^{-1} e^{-i \theta} k\left(\rho e^{i \theta} z\right)=z+\rho^{-2} e^{-2 i \theta} z^{-1}$ $=z+b_{1} z^{-1}$, where $k(z)=z+z^{-1}$. Hence we have $\max _{\Sigma_{0}} \operatorname{Re} L(f)=\operatorname{Re} L(g)$ $=\operatorname{Re} L\left(\rho^{-1} e^{-i \theta} k\left(\rho e^{i \theta} z\right)\right)=\max _{f: \text { odd } \in \Sigma_{0}} \operatorname{Re} L(f)$. Now a ssume that $\sum_{n=0}^{N} \beta_{n} b_{1}^{n} \neq 0$. Then the Gâteaux differential of $L(\cdot)$ at $g$ is given by

$$
l(h)=\left(\sum_{n=0}^{N} \beta_{n} b_{1}^{n}\right) c_{3}+\left(\sum_{m=1}^{M} m \alpha_{m} b_{1}^{m-1}+b_{3}\left(\sum_{n=1}^{N} n \beta_{n} b_{1}^{n-1}\right)\right) c_{1},
$$

$h(z)=z+c_{1} z^{-1}+c_{2} z^{-2}+c_{3} z^{-3}+\cdots$. Thus the omitted set of $w=g(z)$ lies on the trajectory arcs of the quadratic differential

$$
\begin{array}{r}
l\left(\frac{1}{g-w}\right) d w^{2}=\left[\left(\sum_{n=0}^{N} \beta_{n} b_{1}^{n}\right)\left(w^{2}-b_{1}\right)+\left(\sum_{m=1}^{M} m \alpha_{m} b_{1}^{m-1}+b_{3}\left(\sum_{n=1}^{N} n \beta_{n} b_{1}^{n-1}\right)\right)\right] d w^{2} \\
\text { (e.g. [8]). }
\end{array}
$$

Hence we know that $g(z)$ is an odd function by the above theorem. Thus we have the desired result. 
Next we give the real coefficients case.

THEOREM 1.5. Let $f(z)=z+\sum_{n=1}^{\infty} b_{n} z^{-n}$ be in $\Sigma_{0}$. Let $\hat{\boldsymbol{C}}-f(|z|>1)$ be on the trajectory arcs of the quadratic differential $Q(w) d w^{2}=\left(A_{0} w^{2}+A_{1} w+A_{2}\right) d w^{2}$, $\left(A_{0}, A_{1}, A_{2} \in \boldsymbol{R}\right)$. If one of the following conditions is satisfied, then $\overline{f(\bar{z})}=f(z)$.

$$
\begin{array}{ll}
\text { 1) } & A_{0} \geqq 0 \\
\text { 2) } & A_{1}=0 \text { and }\left|A_{2}\right| \geqq 4\left|A_{0}\right| \\
\text { 3) } & A_{2}=0 \text { and }\left|A_{1}\right| \geqq 4\left|A_{0}\right|
\end{array}
$$

Proof. Case 1.1) $A_{0}=0$ and $A_{1}=0$. Then $Q(w) d w^{2}=A_{2} d w^{2}$. It is easy to see that $f(z)=z+z^{-1}$ when $A_{2}>0$ and $f(z)=z-z^{-1}$ when $A_{2}<0$.

Case 1.2) $\quad A_{0}=0$ and $A_{1} \neq 0$. Then $Q(w) d w^{2}=\left(A_{1} w+A_{2}\right) d w^{2}$. By the assump. tion and Schwarz reflection principle we have

$$
\begin{aligned}
& \left(A_{1} f(z)+A_{2}\right) z^{2} f^{\prime}(z)^{2} \\
& \quad=A_{1} z^{3}+A_{2} z^{2}-b_{1} A_{1} z-\left(3 b_{2} A_{1}+2 b_{1} A_{2}\right)-\bar{b}_{1} A_{1} z^{-1}+A_{2} z^{-2}+A_{1} z^{-3} .
\end{aligned}
$$

We put $\phi(w)=\overline{f\left(\overline{\left.f^{-1}(w)\right)}\right.}=w+\left(b_{1}-\bar{b}_{1}\right) w^{-1}+\left(b_{2}-\bar{b}_{2}\right) w^{-2}+\left(b_{3}-\bar{b}_{3}+\bar{b}_{1}\left(b_{1}-\bar{b}_{1}\right)\right) w^{-3}+\cdots$. Applying Lemma 1.1 to the pair of $\phi(w)$ and $\left(A_{1} w+A_{2}\right) d w^{2}$, we have

$$
b_{1}=\bar{b}_{1} \text {. }
$$

Hence the coefficients of the right hand side of (1.3) are real. Comparing the coefficients of both sides of (1.3) we know that all $b_{n}$ are real.

Case 1.3) $A_{0}>0$. By the assumption and Schwarz reflection principle we have

$$
\begin{aligned}
& \left(A_{0} f(z)^{2}+A_{1} f(z)+A_{2}\right) z^{2} f^{\prime}(z)^{2}=A_{0} z^{4}+A_{1} z^{3}+A_{2} z^{2}-\left(2 b_{2} A_{0}+b_{1} A_{1}\right) z \\
& -\left(4 b_{3} A_{0}+3 b_{2} A_{1}+2 b_{1} A_{2}+2 b_{1}^{2} A_{0}\right)-\left(2 \bar{b}_{2} A_{0}+\bar{b}_{1} A_{1}\right) z^{-1}+A_{2} z^{-2}+A_{1} z^{-3}+A_{0} z^{-4} .
\end{aligned}
$$

We denote the right hand side of (1.4) by $z^{-4} q(z)$. Applying Lemma 1.2 to the pair of $\phi(w)$ (see Case 1.2)) and $\left(A_{0} w^{2}+A_{1} w+A_{2}\right) d w^{2}$, the left hand side of (1.2) becomes $\operatorname{Re}\left(A_{0}\left(b_{3}-\bar{b}_{3}\right)+A_{1}\left(b_{2}-\bar{b}_{2}\right)+A_{2}\left(b_{1}-\bar{b}_{1}\right)+(1 / 2) A_{0}\left(b_{1}^{2}-\bar{b}_{1}^{2}\right)\right)=0$. Thus

$$
2 b_{2} A_{0}+b_{1} A_{1}=2 \bar{b}_{2} A_{0}+\bar{b}_{1} A_{1} \text {. }
$$

This means that the coefficients of $q(z)$ are all real. By $w=f(z)(1.4)$ becomes

$$
\left(A_{0} w^{2}+A_{1} w+A_{2}\right) d w^{2}=z^{-6} q(z) d z^{2} .
$$

It follows from this equation that $(-\infty,-1)$ and $(1,+\infty)$, the components of the real axis in $|z|>1$, are mapped by $w=f(z)$ onto trajectory or orthogonal 
trajectory arcs of $\left(A_{0} w^{2}+A_{1} w+A_{2}\right) d w^{2}$ and that $w=f(z)$ is on a trajectory of $\left(A_{0} w^{2}+A_{1} w+A_{2}\right) d w^{2}$ for all sufficiently large real $z$ because $A_{0}>0$. Since the conformal center $\frac{1}{2 \pi} \int_{0}^{2 \pi} f\left(e^{i \theta}\right) d \theta=0$, the omitted set $\Gamma=f(|z|=1)$ must contain the origin. In addition, $f((-\infty,-1))$ and $f((1,+\infty))$ are running from $\Gamma$ to $\infty$. These can be possible only when $f((-\infty,-1))$ and $f(\langle 1,+\infty))$ are on the real axis. Hence $b_{n}$ are all real.

Case 2) $A_{0}<0, \quad A_{1}=0$ and $\left|A_{2}\right| \geqq 4\left|A_{0}\right|$. Then $Q(w) d w^{2}=\left(A_{0} w^{2}+A_{2}\right) d w^{2}$. The distance between the critical points $\pm \sqrt{-A_{2} / A_{0}}$ is $2\left|\sqrt{-A_{2} / A_{0}}\right| \geqq 4$. Moreover, $f(z)$ is odd by Theorem 1.3. Hence it follows that $f(z)=z+z^{-1}$ when $A_{2}>0$ and $f(z)=z-z^{-1}$ when $A_{2}<0$.

Case 3) $A_{0}<0, \quad A_{2}=0$ and $\left|A_{1}\right| \geqq 4\left|A_{0}\right|$. Then $Q(w) d w^{2}=\left(A_{0} w^{2}+A_{1} w\right) d w^{2}$. Since $\frac{1}{2 \pi} \int_{0}^{2 \pi} f\left(e^{i \theta}\right) d \theta=0$ and $\left|-A_{1} / A_{0}\right| \geqq 4$, the omitted set $\Gamma=f(|z|=1)$ contains the origin and does not contain $-A_{1} / A_{0}$. There is $z_{0}$ on the real axis in $|z|>1$ such that $f\left(z_{0}\right)=-A_{1} / A_{0}$ because the right hand side of (1.5) has real coefficients. Let $J$ be one of the components of real axis in $|z|>1$ such that $z_{0} \in J$. Then $f(J)$ is a smooth Jordan arc which is always on trajectory or orthogonal trajectory $\operatorname{arcs}$ of $Q(w) d w^{2}$ and goes from $\Gamma$ to $\infty$ via $-A_{1} / A_{0}$. This can be possible only when $f(J)$ is on the real axis. Hence $b_{n}$ are all real. This completes the proof.

Counterexample. Suppose that $Q(w) d w^{2}=\left(A_{0} w^{2}+A_{2}\right) d w^{2}$ with $A_{0}, A_{2} \in \boldsymbol{R}$, $A_{0}<0$ and $\left|A_{2}\right|<4\left|A_{0}\right|$. Let $\Gamma$ be a continuum symmetric with respect to the origin and not symmetric with respect to the real axis which consists of the segment $\left[-\sqrt{-A_{2} / A_{0}}, \sqrt{-A_{2} / A_{0}}\right]$, which may degenerate, together with the trajectory arcs from $\pm \sqrt{-A_{2} / A_{0}}$. Since the distance between the critical points $\pm \sqrt{-A_{2} / A_{0}}$ is $2 \mid \sqrt{-A_{2} / A_{0}} /<4$, we can take $\Gamma$ with transfinite diameter 1 . Hence there is an odd and not real coefficient function in the class $\Sigma_{0}$ whose omitted set is on the trajectory arcs of the quadratic differential $Q(w) d w^{2}$. The necessity of its oddness is known by Theorem 1.3, too.

\section{Coefficient estimates.}

It is known that the third Ozawa number $B_{3}=3$ by the results of Garabedian and Schiffer [1] and Kirwan and Schober [3]. Now we give its direct proof by making use of Löwner's method (e.g. [7, Chapter 6]).

THEOREM 2.1. 


$$
\operatorname{Max}_{\Sigma_{0}} \operatorname{Re}\left(\lambda b_{1}-b_{3}\right)=\left\{\begin{array}{l}
\lambda \quad \text { for } 3 \leqq \lambda \\
\lambda\left(t_{0}+1\right) e^{-t_{0}}+\frac{1}{2}+\left(\frac{1}{2} t_{0}^{2}-t_{0}-\frac{1}{2}\right) e^{-2 t_{0}} \quad \text { for } 0 \leqq \lambda<3,
\end{array}\right.
$$

where $t_{0}$ is the root of $(3-t) e^{-t}=\lambda$.

Proof. It is sufficient to examine only odd functions in $\Sigma_{0}$ by Corollary 1.4 . If $f(z)=z+b_{1} z^{-1}+b_{3} z^{-3}+\cdots$ is an odd function in $\Sigma_{0}$, then $f\left(z^{-1 / 2}\right)^{-2}=z-2 b_{1} z^{2}$ $-\left(2 b_{3}-3 b_{1}^{2}\right) z^{3}+\cdots$ belongs to the familiar class $S$. We assume that this function has Löwner's coefficient representations, that is to say,

$$
\begin{aligned}
-2 b_{1}= & -2 \int_{0}^{\infty} e^{-t} e^{i \theta(t)} d t \text { and } \\
& -\left(2 b_{3}-3 b_{1}^{2}\right)=-2 \int_{0}^{\infty} e^{-2 t} e^{2 i \theta(t)} d t+4\left(\int_{0}^{\infty} e^{-t} e^{i \theta(t)} d t\right)^{2}
\end{aligned}
$$

where $\theta(t)$ is a continuous function on $(0, \infty)$. Then we have

$$
\begin{aligned}
& \operatorname{Re}\left(\lambda b_{1}-b_{3}\right)= \lambda \int_{0}^{\infty} e^{-t} \cos \theta(t) d t-\int_{0}^{\infty} e^{-2 t} \cos 2 \theta(t) d t \\
&+\frac{1}{2}\left(\left(\int_{0}^{\infty} e^{-t} \cos \theta(t) d t\right)^{2}-\left(\int_{0}^{\infty} e^{-t} \sin \theta(t) d t\right)^{2}\right) \\
& \leqq \lambda \int_{0}^{\infty} e^{-t} \cos \theta(t) d t+\frac{1}{2}-2 \int_{0}^{\infty} e^{-2 t} \cos ^{2} \theta(t) d t+\frac{1}{2}\left(\int_{0}^{\infty} e^{-t} \cos \theta(t) d t\right)^{2} .
\end{aligned}
$$

If we put $\int_{0}^{\infty} e^{-2 t} \cos ^{2} \theta(t) d t=\left(t+\frac{1}{2}\right) e^{-2 t}$ for some $t, 0 \leqq t<\infty$, then it follows from Valiron-Landau Theorem [4] that

$$
\operatorname{Re}\left(\lambda b_{1}-b_{3}\right) \leqq \lambda(t+1) e^{-t}+\frac{1}{2}-2\left(t+\frac{1}{2}\right) e^{-2 t}+\frac{1}{2}(t+1)^{2} e^{-2 t} \equiv \phi(t) .
$$

Then $(d / d t) \phi(t)=t e^{-t}\left((3-t) e^{-t}-\lambda\right)$. Hence $(d / d t) \phi(t) \leqq 0$ for all $t \geqq 0$ if $3 \leqq \lambda$. Thus we have $\operatorname{Re}\left(\lambda b_{1}-b_{3}\right) \leqq \phi(t) \leqq \phi(0)=\lambda$ for $3 \leqq \lambda$. Assume that $0 \leqq \lambda<3$. In this case $\phi(t) \leqq \phi\left(t_{0}\right)$ for $t_{0}$ such that $\left(3-t_{0}\right) e^{-t_{0}}-\lambda=0$. Hence we have

$$
\operatorname{Re}\left(\lambda b_{1}-b_{3}\right) \leqq \lambda\left(t_{0}+1\right) e^{-t_{0}}+\frac{1}{2}+\left(\frac{1}{2} t_{0}^{2}-t_{0}-\frac{1}{2}\right) e^{-2 t_{0}} \quad \text { for } 0 \leqq \lambda<3 .
$$

If we take a piecewise continuous function $\nu(t)$ such that

$$
\cos \nu(t)=\left\{\begin{array}{ll}
e^{t-t_{0}} & \text { for } 0 \leqq t \leqq t_{0} \\
1 & \text { for } t_{0}<t<\infty
\end{array} \text { and } \sin \nu(t)=\left\{\begin{array}{cc}
\left(1-e^{2\left(t-t_{0}\right)}\right)^{1 / 2} & \text { for } 0 \leqq t<t_{1} \\
-\left(1-e^{2\left(t-t_{0}\right)}\right)^{1 / 2} & \text { for } t_{1} \leqq t \leqq t_{0} \\
0 & \text { for } t_{0}<t<\infty
\end{array}\right.\right.
$$


where $t_{1}$ is determined by the condition $\int_{0}^{\infty} e^{-t} \sin \nu(t) d t=0$, then $e^{2 \nu(t)}$ generates a function $h$, which belongs to the class $S$, whose square root inversion transformation $h\left(z^{-2}\right)^{-1 / 2}$ is an extremal function for $\max _{\Sigma_{0}} \operatorname{Re}\left(\lambda b_{1}-b_{3}\right)=\phi\left(t_{0}\right)$. This completes the proof.

Next we give an application of real coefficients case. It is well known that $\left|b_{3}+(1 / 2) b_{1}^{2}\right| \leqq 1 / 2$ and the extremal functions are odd. The oddness of them is found in Corollary 1.4, too. The following estimate complements it in a sense.

THEOREM 2.2. Let $\lambda>0$. Then

$$
\begin{aligned}
& \max \operatorname{Re}\left(b_{3}+\frac{1}{2} b_{1}^{2}+\lambda b_{2}\right)=\frac{17}{864} \lambda^{4}-\frac{4}{27} \lambda^{3}+\frac{2}{9} \lambda^{2}+\frac{8}{27} \lambda+\frac{11}{54} \\
& -\frac{\lambda^{4}}{64} \log \left\{\frac{1}{3 \lambda}\left(\lambda-4+2 \sqrt{\lambda^{2}-2 \lambda+4}\right)\right\}-\left(\frac{17}{864} \lambda^{3}-\frac{7}{72} \lambda^{2}+\frac{1}{9} \lambda-\frac{4}{27}\right) \sqrt{\lambda^{2}-2 \lambda+4} .
\end{aligned}
$$

Extremal function is unique.

Proof. Let $f(z)$ be an extremal function. Then its omitted set $\hat{\boldsymbol{C}}-f(|z|>1)$ is on the trajectory arcs of the quadratic differential $Q(w) d w^{2}=w(w+\lambda) d w^{2}$. So $f(z)$ must have real coefficients by Theorem 1.5 and its omitted set consists of three arcs emanating from the origin. Thus it follows by Schwarz reflection principle that $f(z)(f(z)+\lambda) z^{2} f^{\prime}(z)^{2}$ has double zeros at the points $1, e^{2 \alpha}$ and $e^{-\imath \alpha}$, for some real $\alpha$, which correspond to the three tips, and simple zeros at the points $-r$ and $-r^{-1}$, for some $r>1$, which correspond to the point $-\lambda$. So we can put

$$
\begin{aligned}
& f(z)(f(z)+\lambda) z^{2} f^{\prime}(z)^{2} \\
& =z^{4}+\lambda z^{3}-\left(\lambda b_{1}+2 b_{2}\right) z-2\left(2 b_{3}+b_{1}^{2}+\frac{3}{2} \lambda b_{2}\right)-\left(\lambda b_{1}+2 b_{2}\right) z^{-1}+\lambda z^{-3}+z^{-4} \\
& =z^{-4}\left[(z-1)\left(z-e^{\imath \alpha}\right)\left(z-e^{-\imath \alpha}\right)\right]^{2}(z+r)\left(z+r^{-1}\right)
\end{aligned}
$$

for some real $\alpha$ and $r(r>1)$. A comparison of coefficients gives

$$
\begin{gathered}
\lambda=-4 \cos \alpha+2(R-1), \\
\cos ^{2} \alpha-2(R-1) \cos \alpha-(R-1)=0, \\
\lambda b_{1}+2 b_{2}=-4 \cos \alpha-2(R-1)(2 \cos \alpha+1)(2 \cos \alpha+3) \text { and } \\
2 b_{3}+b_{1}^{2}+\frac{3}{2} \lambda b_{2}=4 \cos ^{2} \alpha+1+2(R-1)\left(4 \cos ^{2} \alpha+4 \cos \alpha+2\right) \\
\text { with } R=\left(r+r^{-1}\right) / 2 .
\end{gathered}
$$

We integrate $(2.1)$ by using the correspondence $0=f(-1)$, 


$$
\int_{0}^{w} \sqrt{w(w+\lambda)} d w=\int_{-1}^{z} z^{-3}(z-1)\left(z-e^{\imath \alpha}\right)\left(z-e^{-\imath \alpha}\right) \sqrt{(z+r)\left(z+r^{-1}\right)} d z .
$$

Then it follows that

$$
\begin{array}{r}
\frac{1}{4}(\lambda+2 w) \sqrt{w(w+\lambda)}+\frac{\lambda^{2}}{8} \log \{(\sqrt{w+\lambda}-\sqrt{w}) /(\sqrt{w+\lambda}+\sqrt{w})\} \\
=F\left(z+\sqrt{(z+r)\left(z+r^{-1}\right)}\right)-F\left(-1+\sqrt{(-1+r)\left(-1+r^{-1}\right)}\right),
\end{array}
$$

where

$$
\begin{aligned}
F(t)= & -\frac{a}{2}(t-1)^{-2}-b(t-1)^{-1}+A \log (t-1) \\
& -\frac{m}{2}(t+1)^{-2}-n(t+1)^{-1}+B \log (t+1) \\
& -\frac{p}{16}(t+R)^{-2}-\frac{q}{4}(t+R)^{-1}+\frac{C}{2} \log (2(t+R))+\frac{t^{2}}{8}+k t
\end{aligned}
$$

with

$$
\begin{aligned}
& a=-(R+1)^{2}, \quad b=\frac{1}{2}(R+1)(4 \cos \alpha-R+1), \quad A=2(R-1) \cos \alpha+\frac{1}{2}(R+1)^{2}-2, \\
& m=(R-1)^{2}, \quad n=\frac{1}{2}(R-1)(4 \cos \alpha-R+3), \quad B=-\frac{1}{2}(R-1)(4 \cos \alpha+R+3), \\
& p=2\left(R^{2}-1\right)^{2}, \quad q=-2\left(R^{2}-1\right)(2 \cos \alpha+1), \quad C=-(R-1)(4 \cos \alpha+R+3)
\end{aligned}
$$

and

$$
k=-\frac{1}{4}(4 \cos \alpha-R+2) .
$$

By (2.3) $\cos ^{2} \alpha=(R-1)(1+2 \cos \alpha)$. Since $R=\left(r+r^{-1}\right) / 2>1$, we have $\cos \alpha>$ $-1 / 2$. Hence it follows from (2.2) and (2.3) that

$$
\cos \alpha=\left(-\lambda-2+\sqrt{\lambda^{2}-2 \lambda+4}\right) / 6 \text { and } R=\left(\lambda+2+2 \sqrt{\lambda^{2}-2 \lambda+4}\right) / 6 .
$$

We substitute $w=z+b_{1} z^{-1}+b_{2} z^{-2}+b_{3} z^{-3}+\cdots$ into the left hand side of (2.6) and expand both sides of it around $z=\infty$. Then we obtain, using (2.7),

$$
b_{1}=-\frac{\lambda^{2}}{12}+\frac{2}{3} \lambda-\frac{1}{3}+\frac{\lambda^{2}}{8} \log \left\{\frac{1}{3 \lambda}\left(\lambda-4+2 \sqrt{\lambda^{2}-2 \lambda+4}\right)\right\}-\frac{1}{3}\left(1-\frac{\lambda}{4}\right) \sqrt{\lambda^{2}-2 \lambda+4}
$$

by comparing the constant terms. By this relation, (2.4), (2.5) and (2.7) we obtain the desired estimate for $b_{3}+(1 / 2) b_{1}^{2}+\lambda b_{2}$. Expanding the left hand side of (2.1), we know that all of the coefficients of $f(z)=z+\sum_{n=1}^{\infty} b_{n} z^{-n}$ are represented in terms of $\lambda$. Thus the extremal function is unique. 


\section{REFERENCES}

[1] Garabedian, P.R. And M. Schiffer, A coefficient inequality for schlicht functions, Ann. of Math. 61 (1955), 116-136.

[2] Jenkins, J.A., On certain extremal problems for the coefficients of univalent functions, J. Analyse Math. 18 (1967), 173-184.

[3] KirWan, W.E., AND G. Schober, New inequalities from old ones, Math. Z. 180 (1982) $19-40$.

[4] LANDAU, E., Über die Blochsche Konstante und zwei verwandte Weltkonstanten, Math. Z. 30 (1929), 608-634.

[5] LeUnG, Y.J. AND G. Schober, Low order coefficient estimates in the class $\Sigma$, Ann. Acad. Sci. Fenn. AI Math. 11 (1986), 39-61.

[6] OzawA, M., Coefficient estimates for the class $\Sigma$, Kodai Math. J. 9 (1986), 123133.

[7] Pommerenke, Ch., Univalent Functions (With a chapter on quadratic differentials by G. Jensen), Vandenhoeck \& Ruprecht in Göttingen, 1975.

[8] Schober, G., Univalent Functions-Selected Topics. Lecture Notes in Mathematics 478, Springer Verlag, Berlin·Heidelberg·New York, 1975.

Department of Mathematics

SCIENCE University OF TOKYO

Noda, Chiba, Japan. 